${ }^{1}$ Health Care and Occupational Medicine Centre 'Med-Alko', Konin

${ }^{2}$ Health Care Centre 'Promed', Konin

${ }^{3} 2^{\text {nd }}$ Department of Internal Medicine, District Hospital, Konin

${ }^{4}$ Department of Infectious and Liver Diseases, Medical University of Lodz

${ }^{5}$ Department of Internal Medicine and Nephrodiabetology, Medical University of Lodz

${ }^{6}$ Department of Nursing with Nursing Practice Laboratories, Chair of Nursing Education, Medical University of Lodz

${ }^{7}$ Department of General and Oncological Gastroenterology, Barlicki University Hospital No. 1, Medical University of Lodz

${ }^{8}$ Department of Diabetology and Internal Diseases, Medical University of Warsaw

\title{
Evaluation of intensified therapeutic education in the prevention of type 2 diabetes in the outpatient care setting
}

\section{ABSTRACT}

Introduction. WHO has recognized diabetes as a noninfectious epidemic disease. Due to the steady increase in the number of diabetic patients, a pandemic of this disease is expected to occur in the coming years. According to WHO estimates from 2016, the incidence of diabetes has quadrupled since 1980 , currently amounting 422 million adults. According to the NATPOL PLUS study, at least 1.6 million Poles suffer from diabetes, and the incidence of type 2 diabetes is estimated at 5-6\% of the entire Polish population. Although type 2 diabetes is considered to be a disease of the middleaged and seniors, the number of diabetic children and adolescents has grown in recent years. The risk factors for type $\mathbf{2}$ diabetes are commonly known and can be easily identified in the population. The research objective was to evaluate how effective selected therapeutic education methods provided for obese people are in the prevention of type 2 diabetes. Another aim was to assess the impact of such education on certain anthropometric and laboratory parameters in outpatient health care conditions.

Material and methods. The study included 50 individuals who met the following inclusion criteria: age $\geq \mathbf{3 0}$,

Address for correspondence:

prof. dr hab. n. med. Leszek Czupryniak

Klinika Diabetologii i Chorób Wewnętrznych WUM

ul. Banacha 1a, 02-097 Warszawa

Phone: +48225992583

e-mail: leszek.czupryniak@wum.edu.pl

Translation: lek. Małgorzata Kamińska

Clinical Diabetology 2017, 6, 2, 57-64

DOI: $10.5603 /$ DK.2017.0010

Received: 14.05.2017

Accepted: 01.06.2017
BMI $\geq 30$, and no known blood glucose disorders reported in the interview. The participants were divided into 2 education groups: the intensive Group 1, subjected to education in the cycle of 0-1-2-3 months (four doctor's appointments), and the standard Group 2, educated in the cycle of 0-3 months (two doctor's appointments). For each participant, the following measurements were taken: height, body weight, and waist circumference. Also, each participant was subjected to two $75 \mathrm{~g}$ oral glucose tolerance tests (OGTT) with blood samples drawn at 0 and $120 \mathrm{~min}$ : one at the beginning of the intervention and another 3 months after receiving first health recommendations. Plasma insulin in OGTT and HOMA-IR index were also assessed. Results. Patients from Group 1 reduced their body weight by $9.6 \pm 5.5 \mathrm{~kg}$, whereas in the case of Group 2 it was $3.6 \pm 6.0 \mathrm{~kg}(p<0.01)$. The percentage of patients with normal/abnormal glucose tolerance changed from $29 / 71$ to $58 / 42$ in Group 1, and from $18 / 82$ to $32 / 68$ in Group 2 ( $p<0.05)$.

Conclusions. By increasing the number of medical appointments focused on healthy lifestyle advice in type 2 diabetes prevention in obese outpatients from two to four, it is possible to achieve more effective weight loss, to improve metabolic parameters, and to normalize glucose disorders. (Clin Diabetol 2017; 6, 2: 57-64)

Key words: diabetes prevention, obesity, education

\section{Introduction}

Diabetes is the first disease recognized by the World Health Organization (WHO) as non-infectious 
global epidemic. We are currently observing a steady increase in the incidence of diabetes, and WHO estimates that this increase is pandemic. According to the International Diabetes Federation (IDF), 366 million people worldwide have diabetes, and it is projected that by 2030 the number of diabetics will almost double to reach 551 million [1]. Previous predictions by WHO (1998) indicated that by 2025 , the number of diabetic patients in the world would reach 300 million. However, the rate of increase in the incidence of diabetes in recent years has exceeded expectations and forecasts. According to WHO estimates from 2016, there are currently 422 million adults suffering from diabetes [2].

In Poland diabetes affects about 3 million people. A study conducted in Poland by Wittek et al. showed that the prevalence of diabetes was approximately $8 \%$ [3]. The authors suggest that $5.56 \%$ of Polish population are diagnosed with diabetes and in $2.25 \%$ diabetes remains undiagnosed. The results achieved by the researchers are consistent with the data reported by IDF $[1,3]$. The Screen-Pol study revealed that type 2 diabetes affects $12-15 \%$ of patients over 40 years of age reporting to primary care physicians [4].

Diabetes risk factors are known and easy to identify in the population. They can be divided into two groups: genetic predispositions and environmental and constitutional factors. The first group of risk factors includes a family history of diabetes - the risk of developing the disease increases to $15 \%$, if one parent is diabetic, and if both parents have diabetes, the risk is as high as $75 \%$ [4]. Type 2 diabetes is a multigene disorder, and its genetic background seems to be very complex. Today, it is not possible, based on genetic studies, to precisely define a group that will develop type 2 diabetes in the future, so we cannot focus on the individualization of treatment based on genetic profile. For this reason, in prevention of type 2 diabetes in people at risk special emphasis should be placed on environmental and constitutional factors such as weight reduction, exercise and change of dietary habits.

The main environmental factor responsible for the rapid rise in diabetes incidence is abdominal obesity. An increase in the incidence of type 2 diabetes is always preceded by an increase in the number of obese people. According to $\mathrm{WHO}$, overweight and obesity are responsible for $80 \%$ of type 2 diabetes $[2,5]$.

An environmental factor closely related to the development of type 2 diabetes is nutrition. It has been shown that a low-fibre diet containing high amounts of carbohydrates and fats increases the risk of developing this type of diabetes. In addition, lack of regular exercise results in obesity and consequently type 2 diabetes [4].
Polish guidelines on the management of diabetes recommend active search for metabolic disorders. Patients at risk should be screened for diabetes, because the symptoms of hyperglycaemia are absent in more than half of them. Screening tests for diabetes should be performed every 3 years in all patients over 45 years of age and once a year in patients at risk [6].

Considering the epidemic of diabetes and the fact that, according to the Polish Guidelines, all preventive actions aimed at stopping this epidemic (e.g. healthy lifestyle promotion) should be performed at the level of primary health care, we designed this study to determine whether diabetes prevention can be carried out in the current working conditions of the primary care facilities. The research objective was to evaluate how effective selected therapeutic education methods provided for obese people are in the prevention of type 2 diabetes and to assess the impact of such education on certain anthropometric and laboratory parameters in outpatient health care conditions.

\section{Patients}

The study was conducted in one health care facility - the family physician centre in Konin. The study included 50 consecutive patients who met the inclusion criteria and declared the intention of weight reduction under the control of a physician and who gave their written consent for participation in the study after reading the study protocol. The study was a prospective randomized clinical trial, with a follow-up period of 3 months. The subjects were randomly divided into two groups according to intensity of care expressed by the frequency of doctor's appointments:

- a group offered a standard outpatient care the frequency of doctor's visits was 3 months (at baseline and after 3 months); the cycle of $0-3$ months;

- a group offered an intensive outpatient care doctor' visits were scheduled every month, a total of four visits (every month for 3 consecutive months), the cycle of $0-1-2-3$ months.

The groups differed only in the frequency of visits. During each visit, the patients were provided with education and, if appropriate, therapeutic treatment. Eligibility criteria for the study were age over 30 years, $\mathrm{BMI} \geq 30 \mathrm{~kg} / \mathrm{m}^{2}$, and no known history of glucose metabolism disorders. Patients with diabetes or other disorders of glucose metabolism, or who have taken drugs with known hyperglycaemic effect (e.g. steroid hormones), those with gastrointestinal disease, or other conditions that impede the performance of OGTT, patients receiving metformin for any indication other than carbohydrate disorders (e.g. polycystic ovary 
syndrome) and those with cancer (diagnosed less than 5 years ago) were excluded from the study.

\section{Methods}

The study protocol was approved by the Bioethics Committee of the Medical University of Lodz. All the patients gave written consent to participate in the study after reading the patient information. Eligible patients who agreed to participate in the study were randomized into two groups after initial therapeutic education.

In all patients, a medical interview and physical examination were performed, taking into account inclusion and exclusion criteria. The following measurement were taken: height; body weight to the nearest of $0.1 \mathrm{~kg}$, waist circumference measured with a measuring tape, directly on the patient's skin, to the nearest of $1 \mathrm{~cm}$, midway between the lower edge of the rib arch and iliac crest; hip circumference measured with an inelastic measuring tape to the nearest of $1 \mathrm{~cm}$, at the level of the most prominent buttock muscle. The $\mathrm{BMI}$ was calculated according to the formula: $\mathrm{BMI}=$ body weight in $\mathrm{kg} /(\text { height in } \mathrm{m})^{2}$. Blood pressure was measured in a doctor's office by the Korotkov method using an Accoson mercury manometer, with a patient in a sitting position with a shoulder at heart level, after a 15 -minute rest.

All patients underwent a 75-g OGTT with blood sampling at 0 and 120 min twice: at baseline and at 3 months after the first health recommendations had been given. The patients were asked to drink a solution of $75 \mathrm{~g}$ of glucose dissolved in $300 \mathrm{~mL}$ of water within 2-3 minutes. During the test, the patients did not exercise, drink fluids, nor eat any meals. The OGTT was performed after 8 to 12 hours of fasting. The first blood samples were taken around $8 \mathrm{am}$. Glucose levels were determined immediately after blood collection and blood serum for insulin level determination was frozen at $-82^{\circ} \mathrm{C}$ (SANYO freezer, Japan) and assigned the serial numbers at a later time.

Based on fasting glucose and insulin concentrations, the HOMA-IR insulin resistance index was calculated according to the following formula: HOMA-IR = fasting insulin $(\mu \mathrm{U} / \mathrm{mL}) \times$ fasting glucose $(\mathrm{mmol} / \mathrm{L}) / 22.5$ [7].

After qualifying for the study, the patients were randomized using a randomization calculator developed by the Social Psychology Network (www.randomizer.org). Each of the 50 patients included in the study was assigned to one of two groups - standard or intensive. Dietary recommendations were identical for all subjects, the groups differed only in frequency of visits. Qualification for study and Visit 0 were made during the same visit to the clinic, as the inclusion and exclusion criteria were based only on medical history data and did not require laboratory testing and interpretation of their results. This approach is closest to the everyday practice of a family doctor.

At every visit (two doctor's visits during 3 months in the standard group and four doctor's visit in the intensive group) education was provided for the patients on lifestyle modifications. This included simple dietary recommendations in oral and written form. The main emphasis was put on the avoidance of mono- and disaccharides. Patients were advised to avoid fruits and their derivatives in the form of juices, jams, and compotes. Fruits in the diet were to be replaced with vegetables. During the educational session, the table included in the textbook "Diabetes" by prof. Sieradzki was used, but modified by eliminating fruits and increasing the number of vegetables to seven servings [8].

The daily ration was: 5 servings of cereal products, 2 servings of dairy products, 1 serving of meat or its substitutes, 2 servings of fat, 7 servings of vegetables. The basic daily ration (about 1,500 calories) could be increased by the patient according to his/her own needs and instructions given. Each patient also received written general dietary recommendations, a description of nutritional servings and recipes for preparing meals.

As mentioned above, patients in the intensive group were provided with the same education every month. The patients were given the same recommendations and the same educational materials were used as at first visit. The patients who lost the written recommendations received another one.

Each patient was recommended a minimum of 30 minutes of physical exercise three times a week, but the patients were encouraged to exercise every day to achieve better results. Walking or Nordic walking was suggested, but any aerobic exercise of specified duration was acceptable. It was emphasized that this exercise should be added to current physical activity. Moreover, it was recommended that physical activity be continuous, and the patients were not allowed to divide it into shorter periods, e.g. two 15-minute cycles per day. The exercise was expected to be intensive enough to raise the heart rate above $120-130$ beats per minute.

As mentioned above, the patients in the intensive group received repeated recommendations for physical activity every month up to 3 months after their first "educational" contact with a physician.

The obtained results were statistically analysed with Statistica 10.1 PL software. The Shapiro-Wilk test was used to assess the normality of data distribution. For the assessment of between-group differences in normally distributed variables, the Student's t-test for independent samples was used, and to evaluate the 
Table 1. Characteristics of the study groups

\begin{tabular}{|c|c|c|c|}
\hline & All study participants & Standard group & Intensive group \\
\hline $\mathrm{N}$ & 46 & 22 & 24 \\
\hline Age (years) & $53 \pm 12$ & $53 \pm 12$ & $53 \pm 11$ \\
\hline Body weight $[\mathrm{kg}]$ & $94.2 \pm 17.6$ & $90.9 \pm 14.6$ & $99.3 \pm 20.8$ \\
\hline Height $[\mathrm{cm}]$ & $165 \pm 9.4$ & $163 \pm 7.9$ & $169 \pm 10.8$ \\
\hline $\mathrm{BMI}\left[\mathrm{kg} / \mathrm{m}^{2}\right]$ & $34.3 \pm 3.9$ & $34.09 \pm 3.6$ & $34.7 \pm 3.4$ \\
\hline Waist circumference $(\mathrm{cm})$ & $104.8 \pm 13.2$ & $103 \pm 12.2$ & $106.9 \pm 14.9$ \\
\hline Hip circumference $[\mathrm{cm}]$ & $114.2 \pm 10.1$ & $113.5 \pm 10.2$ & $115.1 \pm 10.2$ \\
\hline Systolic blood pressure [mm Hg] & $134.2 \pm 10$ & $134.5 \pm 11$ & $133.9 \pm 10$ \\
\hline Diastolic blood pressure [mm Hg] & $82.8 \pm 10.4$ & $81.6 \pm 10$ & $84.7 \pm 10.9$ \\
\hline
\end{tabular}

Data are presented as mean and standard deviation (SD)

Table 2. Results of body weight, waist circumference, hips circumference and BMI measurements. Statistical significance regards dependent differences (within a given group)

\begin{tabular}{|c|c|c|c|c|c|c|}
\hline & \multicolumn{3}{|c|}{ Standard group $(\mathrm{n}=22)$} & \multicolumn{3}{|c|}{ Intensive group $(n=24)$} \\
\hline & At 0 months & At 3 months & p-value & At 0 months & At 3 months & p-value \\
\hline Body weight $[\mathrm{kg}]$ & $90.9 \pm 14.6$ & $87.3 \pm 14.0$ & $p=0.0003$ & $99.3 \pm 20.8$ & $89.7 \pm 20.0$ & $p=0.0003$ \\
\hline BMI $\left[\mathrm{kg} / \mathrm{m}^{2}\right]$ & $34.09 \pm 4.6$ & $32.7 \pm 4.8$ & $p=0.0002$ & $34.7 \pm 5.4$ & $31.3 \pm 5.3$ & $p=0.0002$ \\
\hline Waist circumference $[\mathrm{cm}]$ & $103 \pm 12.2$ & $96.8 \pm 10.4$ & $p=0.0001$ & $106.9 \pm 14.9$ & $94.5 \pm 14.6$ & $p=0.0003$ \\
\hline Hip circumference [cm] & $113.5 \pm 10.2$ & $109.8 \pm 8.9$ & $p=0.0003$ & $115.1 \pm 10.2$ & $107.8 \pm 11.1$ & $p=0.0004$ \\
\hline
\end{tabular}

parameters in the same group, Student's t-test for dependent samples was utilized. The U-Mann-Whitney test was used for non-normally distributed variables. Statistical significance was assumed at $p<0.05$. The significance of the relationship between quantitative variables was assessed based on the correlation analysis (Pearson coefficient).

\section{Results}

The study included 50 patients of a family doctor clinic in Konin who were obese and had no history of known glycaemic disorders. The subjects were randomly divided into two groups: a group provided with intensive education (intensive group) and a group provided with standard education (standard group).

Four patients were excluded from the study because they were diagnosed with diabetes based on the assessment performed at Visit 0 , clinical symptoms and/or random blood glucose measurement during the first weeks of the study. Finally, data was collected for 46 people. The detailed characteristics of the analysed groups are shown in Table 1. These parameters did not differ significantly between the standard and intensive groups.

Anthropometric measurements showed that baseline body weight was $8.4 \mathrm{~kg}$ lower in the standard group than in the intensive group, but the BMI was not statistically significantly different between groups. Statistically significant reduction in body weight was observed in both group. In the standard group it was $3.6 \pm 6.0 \mathrm{~kg}$, whereas in the intensive group the weight reduction was 2.5-fold higher and amounted to $9.6 \pm 5.5 \mathrm{~kg}$. In the standard group, the waist circumference was reduced by $6.5 \pm 6.8 \mathrm{~cm}$ and the hip circumference - by $3.7 \pm 4.6 \mathrm{~cm}$. In the intensive group the waist circumference was reduced by $12.3 \pm 6.5 \mathrm{~cm}$ and the hip circumference - by 7.3 $\pm 6.4 \mathrm{~cm}$. The differences between baseline and final values were statistically significant in both group, with a significantly greater reduction in waist circumference in the intensive group compared with the standard group ( $p<0.05$, between-group comparison). The BMI in the standard group decreased by $1.3 \pm 2.0 \mathrm{~kg} / \mathrm{m}^{2}$ and in the intensive group by $3.3 \pm 1.9 \mathrm{~kg} / \mathrm{m}^{2}(p<0.001$ for each group). Weight, waist circumference, hip circumference and BMI measurements as well as p-values are given in Table 2 .

All patients underwent a 2-hour OGTT test according to the WHO protocol and the Polish guidelines on the management of diabetes [6]. The results were interpreted in accordance with the recommendations outlined in the Introduction. During the Visit 0, a total of 35 patients had abnormal OGTT results, i.e. impaired glucose tolerance (IGT) and/or impaired fasting glucose 
Table 3. The results of the oral glucose tolerance test in the studied groups

\begin{tabular}{|c|c|c|c|c|}
\hline & \multicolumn{2}{|c|}{ Standard group $(n=22)$} & \multicolumn{2}{|c|}{ Intensive group $(n=24)$} \\
\hline & At 0 months & At 3 months & At 0 months & At 3 months \\
\hline \multirow[t]{2}{*}{ Normal glucose tolerance } & 4 & 7 & 7 & 14 \\
\hline & $(18 \%)$ & $(32 \%)$ & $(29 \%)$ & $(58 \%)$ \\
\hline \multirow[t]{2}{*}{ Glucose intolerance (IFG i IGT) } & 18 & 15 & 17 & 10 \\
\hline & $(82 \%)$ & $(68 \%)$ & $(71 \%)$ & $(42 \%)$ \\
\hline \multirow[t]{2}{*}{ IFG } & 14 & 14 & 9 & 8 \\
\hline & $(64 \%)$ & $(64 \%)$ & $(38 \%)$ & $(33 \%)$ \\
\hline \multirow[t]{2}{*}{ IGT } & 4 & 1 & 8 & 2 \\
\hline & (18\%) & (4\%) & $(33 \%)$ & $(8 \%)$ \\
\hline
\end{tabular}

Percentages calculated in relation to overall number of patients in a given group IFG — impaired fasting glucose; IGT — impaired glucose tolerance

(IFG): 18 in the standard group and 17 in the intensive group. After 3 months of intervention, disturbances of glucose metabolism were observed in 15 subjects in the standard group and in 10 subjects in the intensive group.

At baseline, 14 patients in the standard group had IFG, 4 patients had IGT and 5 patients had both IFG and IGT. After 3 months, the number of patients with IFG did not change in this group and amounted to 14 , the number of patient with IGT decreased to 1 and there remained 4 patients with coexistence of IFG and IGT. Normalization of glucose metabolism was observed in 7 patients who had IFG at baseline and in 1 patient with IGT (the improvement was observed in a total of 8 patients). There were 5 patients with newly diagnosed glucose metabolism disorders - IFG was diagnosed de novo in 4 patients and IGT in 1 patient. Overall, after 3 months of intervention, the number of patients with normal glucose tolerance in the standard group increased by 3 .

In the intensive group, IFG was diagnosed during the Visit 0 in 9 patients, IGT in 8 patients, IFG + IGT in 5 patients. After 3 months of intervention, 8 patients still had IFG, IGT was found in 2 patients and both disorders (IFG and IGT) were present in 5 patients. There weren't any new carbohydrate disorders in this group. In both groups, weight reduction was most associated with a decrease in the prevalence of IGT.

After 3 month, the normalization of OGTT results was found in 10 patients in both groups, which accounts for $22 \%$ of the overall study group. The number of patients with diagnosed glucose tolerance abnormalities was decreased by $14 \%$ in the standard group and by $29 \%$ in the intensive group; the differences were statistically significant $(p<0.05)$.

Relative risk reduction of any type of glucose intolerance was 0.17 in the standard group and 0.41 in the intensive group, and was significantly (2.4 times) higher in the latter group than in the first group. The results for both groups are presented in Table 3.

At baseline, fasting glucose concentration in the intensive group was $10.3 \mathrm{mg} / \mathrm{dL}$ higher on average compared to the standard group. After 3 months, both groups did not differ in this regard, whereas only in the intensive group a statistically significant $(p=0.03$ ) reduction in mean fasting plasma glucose was obtained.

The mean blood glucose at 2 hours of the $75-\mathrm{g}$ OGTT decreased by $14.0 \mathrm{mg} / \mathrm{dL}$ in the standard group, but no statistically significant difference was obtained ( $p=0.3$ ). In the intensive group, a statistically significant decrease in blood glucose at this time-point was achieved and it amounted $33.7 \mathrm{mg} / \mathrm{dL}$ on average ( $p=0.02)$.

At 0 and 120 minutes of OGTT serum insulin concentration was measured. Prior to study intervention, during Visit 0 , higher values of fasting insulin were observed in the standard group compared to the control group $(30.73 \pm 18.8$ vs. $20.35 \pm 13.6 \mu \mathrm{lU} / \mathrm{mL})$, whereas insulin concentrations at 2 hours of OGTT were similar in both groups (85.02 \pm 54.01 vs. 84.34 $\pm 68.9 \mu \mathrm{lU} / \mathrm{mL}$ ). Insulin levels, both in fasting state and 2 hours after glucose challenge, were decreased after intervention, and were similar in both groups, with a statistically significant change from baseline values measured at Visit $0(p<0.05)$.

The insulin resistance index HOMA-IR was also evaluated. A reduction of this parameter was obtained after intervention in both groups. In the standard group, the mean HOMA-IR at baseline (before the intervention) was $7.76 \pm 5.19$ vs. $3.01 \pm 2.22$ after intervention, while in the intensive group respective values were $6.12 \pm 4.58$ vs. $2.34 \pm 2.98$. The reduction of HOMA-IR was statistically significant in both groups ( $p<0.01)$. The results of biochemical test are presented in Table 4. 
Table 4. Blood glucose and insulinaemia during oral glucose tolerance test (OGTT) and insulin resistance index (HOMA-IR)

\begin{tabular}{|c|c|c|c|c|c|c|}
\hline & \multicolumn{3}{|c|}{ Standard group $(n=22)$} & \multicolumn{3}{|c|}{ Intensive group $(\mathrm{n}=24)$} \\
\hline & $\begin{array}{c}\text { At } 0 \\
\text { months }\end{array}$ & $\begin{array}{c}\text { At } 3 \\
\text { months }\end{array}$ & $\begin{array}{c}\text { Statistical } \\
\text { significance }\end{array}$ & $\begin{array}{c}\text { At } 0 \\
\text { months }\end{array}$ & $\begin{array}{c}\text { At } 3 \\
\text { months }\end{array}$ & $\begin{array}{c}\text { Statistical } \\
\text { significance }\end{array}$ \\
\hline Fasting blood glucose [mg/dL] & $105.5 \pm 18.2$ & $104.9 \pm 15.2$ & $p=0.50$ & $115.8 \pm 17.4$ & $105.2 \pm 18.0$ & $p=0.03$ \\
\hline Glucose at 2 h OGTT [mg/dL] & $149.1 \pm 68.1$ & $129.5 \pm 45.71$ & $p=0.32$ & $161.3 \pm 60.7$ & $131.3 \pm 44.1$ & $p=0.02$ \\
\hline Fasting insulin $[\mu \mathrm{IU} / \mathrm{mL}]$ & $30.73 \pm 18.8$ & $11.72 \pm 8.62$ & $p=0.001$ & $20.35 \pm 13.6$ & $10.72 \pm 12.44$ & $p=0.01$ \\
\hline Insulin at $2 \mathrm{~h}$ OGTT $[\mu \mathrm{IU} / \mathrm{mL}]$ & $85.02 \pm 54.01$ & $53.55 \pm 48.7$ & $p=0.04$ & $84.34 \pm 68.9$ & $48.50 \pm 30.59$ & $p=0.02$ \\
\hline HOMA-IR & $7.76 \pm 5.19$ & $3.01 \pm 2.22$ & $p=0.001$ & $6.12 \pm 4.58$ & $2.34 \pm 2.98$ & $p=0.004$ \\
\hline
\end{tabular}

\section{Discussion}

Type 2 diabetes is the most common form of diabetes. The main cause of diabetes and obesity, two disturbances that are the background for cardiovascular disease, is unhealthy lifestyle, including poor diet. In the Diabetes Prevention Program (DPP) lifestyle changes (diet, physical activity, and weight reduction) decreased the risk of developing type 2 diabetes by 58\% [9]. Similar results were obtained in the Finnish Diabetes Prevention Study (DPS) [10].

Studies show that diabetes can be prevented in large part, but the problem is translating the results of large clinical trials into everyday practice. Our study was performed to answer the question: Whether this model of therapeutic education would be effective in outpatient health care setting in Poland and which method of education would provide better results? The average patient finds it difficult to maintain the discipline to consistently implement lifestyle changes, therefore a time limit of 3 months was set for implementing the education programme. Education was carried out individually while providing medical care in the family doctor clinic.

Due to the fact that the study was planned to be carried out in outpatient setting, the time restrictions and great diversity of patient (different levels of education, culinary skills, motivation) had to be taken into account; therefore, efforts were made to simplify the recommendations as much as possible, so that they can be easily understood and remembered by patients. In brief, pathophysiological mechanisms of basic dietary errors were described to the patients and it was explained why a specific recommendation is beneficial for weight reduction. During the training of each patient, visualization was performed on materials intended to be taken home.

The recommendations given to the patients included an example of a weekly diet with specific recipes developed mainly by the person who delivered the educational intervention as well as suggestions of professional nutrition guides [11]. The recipes were simple, so that preparing the meals was not time-consuming.
The patients could cook more food to have ready meals for a couple of days. The recipes did not include fruit ingredients. Particular attention was paid to the terminology used, avoiding professional medical terms.

In the study group, the emphasis was placed on avoiding free sugars. According to WHO/FAO 1998 definition, free sugars include monosaccharides, such as glucose, fructose, galactose or maltose, and disaccharides, such as sucrose, maltose or lactose. Recently, the role of sugars added to food in the development of obesity and diabetes and its adverse effects on cardiovascular risk factors have been raised, hence the assumption that this group of the nutrients should be avoided [12-14]. The added sugars are defined as the sugar or syrup that is added to processed food and table sugar. Added sugars include white sugar, brown sugar, honey, maple syrup, corn syrup and dextrose, sugar contained in sweetened beverages, including fruit juices. It was calculated that the addition of added sugars as an extra daily portion of the drink (150 kcal) to the current diet would increase the body weight by $6.75 \mathrm{~kg}$ a year [15]. In the Nurses' Health Study II, it was observed that a group consuming more than 1-2 servings of sweetened beverages daily had an $83 \%$ higher risk of type 2 diabetes than those consuming such beverages less than once a month [16]. In view of the above, the dietary education of patients especially stressed the avoidance of fruits and their derivatives in the form of juices, compotes, jams, jellies and marmalades, even those referred to as low-sugar. It was recommended that these nutrients should be replaced by vegetables, thereby increasing the amount of fibre in the diet. Elimination of as much sugar as possible from the diet resulted in weight reduction in both groups, with the intensive group achieving two and a half times better weight-loss results: $-3.6 \mathrm{~kg}$ vs. $9.6 \mathrm{~kg}$ $(p=0.0003)$. In the standard group, the reduction was $3.9 \%$ of baseline body weight, while in the intensive group it was $9.6 \%$ of baseline body weight. The resulting weight loss was primarily associated with a reduction in the incidence of IGT. These results are 
similar to data available in the literature where during 16 to 50 -week intervention resulted in a reduction in baseline body weight by $8.0-9.7 \%$, but none of these studies evaluated diets limiting primarily the amount of free sugars [17-20].

The assumption that the best strategy is to eliminate free sugars from the diet proved to be effective, both in the standard group offered a single educational visit and in the intensive group patients who attended three educational visits.

The prevalence of abnormal glucose tolerance in the study group was almost ten times higher than in the general adult population in Poland: glucose metabolism disturbances were found in $76 \%$ of study participants, while the literature data reported glucose metabolism disorders in $8.44 \%$ of the general adult population [6]. In another well-known interventional trial, the Diabetes Prevention Program (DPP), evaluating the impact of lifestyle changes on the prevention of type 2 diabetes, $33 \%$ of the subjects had IFG [4]. Relatively high percentage of glucose tolerance disorders among study participants was closely related to the mean age of $53 \pm 12$ years of the evaluated group, and age is a postulated factor contributing to the development of type 2 diabetes. Additionally, obesity has been present in each patient (BMI above $30 \mathrm{~kg} / \mathrm{m}^{2}$ was an inclusion criterion).

Following the implementation of dietary intervention, normalization of glucose tolerance was achieved in $21 \%$ of patients in both groups, with a $29 \%$ decrease in the intensive group and $14 \%$ decrease in the standard group. Among placebo-treated patients in the STOP-NIDDM trial assessing behavioural therapy, $31 \%$ of subjects [21] had normal glucose tolerance; therefore, it can be stated that the assumptions of our study, despite short-term follow-up, are appropriate and equally effective. In the DPP $85.6 \%$ of patients in the lifestyle modification group did not develop diabetes at 3 years of follow-up. Perhaps the longer duration of the intervention, the better results achieved by patients [9]

Blood glucose at fasting state and after 2 hours of OGTT decreased in both groups, but only in the intensive group the observed changes were statistically significant $(p<0.05)$. The results obtained in our study for fasting and 2-hour postload blood glucose (-9.3 and $-33.7 \mathrm{mg} / \mathrm{dL}$, respectively) are better than those reported in DPS trial ( -3.6 and $-16.2 \mathrm{mg} / \mathrm{dL}$, respectively), but the assessed intervention was short-term, whereas in the DPS trial, the first results were announced one year after the onset of lifestyle modification [10].

One of the parameters evaluated in our study was the HOMA-IR insulin resistance index, calculated according to the formula developed by Matthews et al., based on values obtained with a highly-correlating method of normoglycaemic clamp developed by Ralph DeFronzo [22], which is considered as the gold standard for the assessment of insulin resistance. Dietary intervention positively influenced the parameters of insulin resistance in both groups. There was a statistically significant decrease in insulinaemia at fasting state and 2 hours post glucose challenge in both the standard and the intensive group. After decreasing the consumption of free sugars and increasing physical activity, significant improvements in insulin action, decreased insulin resistance and reduced excessive compensatory secretion of insulin both in fasting state and 2 hours after oral glucose challenge were observed. In addition, fasting GLP-1 has been reported to improve, with no significant change in glucagon level in fasting state and at 2 hours of OGTT.

According to previous studies, each lifestyle intervention has measurable effects in the prevention of type 2 diabetes. The choice of a method of education is not important, because in each of the large trials evaluating the prevention of diabetes, satisfactory results were obtained. In the DPS trial, a $58 \%$ relative risk reduction of diabetes was achieved in the study group by reducing the supply of calories from fats $<30 \%$ of diet energy, reducing the supply of saturated fatty acids $<10 \%$ of diet energy, increasing fibre supply to $>15 \mathrm{~g} / 1000 \mathrm{kcal}$ and performing moderate physical exercise - at least $30 \mathrm{~min} / 24 \mathrm{~h}$ [10]. Low-fat and low-cholesterol diets along with physical exercise for at least 150 minutes a week were recommended in the DPP and a $58 \%$ reduction in the risk of diabetes was [9]. The anthropometric parameters (body mass, waist and hip circumference) as well as laboratory values were improved in the examined group as a consequence of significant reduction of free sugars and replacing them with complex carbohydrates and physical exercise of at least 30 minutes 3 times a week. The intensive group achieved better results for all assessed parameters.

In our study, the increase in the number of control visits during weight loss induced better outcomes; however, even standard education resulted in favourable changes in body mass, BMI, waist circumference, hip circumference, HOMA-IR, and fasting and 2 hourOGTT insulin levels.

No intermediate targets were recommended in the instructions given to the study participants. In the intensive group, control visits were aimed at repeating the same recommendations as in the initial visit. No anthropometric measurements were made during monthly study visits, only education on diet and exercise was continued. A diet based on natural products 
with a low content of free sugars met the nutritional needs of the patients and promoted satiety. In addition, it enabled the planning of varied meals and allowed continuing such nutrition indefinitely.

Glucose levels measured in fasting state and at 2 hours of OGTT confirm the assumption that free sugars should be limited or eliminated from the diet of obese people at risk of developing type 2 diabetes.

The results show that two-fold increase in the frequency of doctor's visits, during which therapeutic education is offered, by shortening the interval between them from 3 months to 1 month, leads over three months to three-times higher weight reduction and almost two times higher reduction in the occurrence of carbohydrate tolerance disorders compared to the standard frequency of visits i.e. every 3 months. The final conclusion from the study is that prevention of diabetes is possible in outpatient primary care setting; it only requires much more time than it is under standard care conditions.

\section{REFERENCES}

1. IDF Diabetes Atlas. 2011; 12: 06

2. WHO Press Release. 1998; 09: 14.

3. Wittek A, Sokalski B, Grzeszczak W, et al. Prevalence of diabetes and cardiovascular risk factors of industrial area in southern Poland. Exp Clin Endocrinol Diabetes. 2009; 117(7): 350-353, doi: 10.1055/s-0029-1220689, indexed in Pubmed: 19536734.

4. Strojek K. Diabetologia, Termedia Poznań 2014. str : 9-28.

5. The First WHO Global report on Diabetes. ; 2016

6. Polskie Towarzystwo Diabetologiczne. Zalecenia kliniczne dotyczące postępowania z chorymi na cukrzycę. Stanowisko Polskiego Towarzystwa Diabetologicznego. Clin Diabetol. 2017; 6(supl. A): A5-A6.

7. Matthews DR, Hosker JP, Rudenski AS, et al. Homeostasis model assessment: insulin resistance and beta-cell function from fasting plasma glucose and insulin concentrations in man. Diabetologia. 1985; 28(7): 412-419, indexed in Pubmed: 3899825.

8. Sieradzki J. (red.). Cukrzyca, Via Medica, Gdańsk 2007.

9. Knowler WC, Barrett-Connor E, Fowler SE, et al. Diabetes Prevention Program Research Group. Reduction in the incidence of type 2 diabetes with lifestyle intervention or metformin. N Engl J Med. 2002; 346(6): 393-403, doi: 10.1056/NEJMoa012512, indexed in Pubmed: 11832527.

10. Tuomilehto J, Lindstrom F, Briksson J, et al. For the Finnish Diabetes Prevention Study Group. Prevention of type 2 diabetes mellitus by change in lifestyle among subject with impaired glucose tolerance. N Engl J Med. 2001; 344: 1343-1350.

11. Cichocka A. Praktyczny poradnik żywieniowy w odchudzaniu oraz profilaktyce i leczeniu cukrzycy typu 2. Medyk Sp. z o.o., Warszawa; 2010.

12. Vartanian LR, Schwartz MB, Brownell KD. Effects of soft drink consumption on nutrition and health: a systematic review and meta-analysis. Am J Public Health. 2007; 97(4): 667-675, doi: 10.2105/AJPH.2005.083782, indexed in Pubmed: 17329656.

13. Johnson RK, Appel $\amalg$, Brands M, et al. American Heart Association Nutrition Committee of the Council on Nutrition, Physical Activity, and Metabolism and the Council on Epidemiology and Prevention, American Heart Association Nutrition Committee of the Council on Nutrition, Physical Activity, and Metabolism and the Council on Epidemiology and Prevention. Dietary sugars intake and cardiovascular health: a scientific statement from the American Heart Association. Circulation. 2009; 120(11): 1011-1020, doi: 10.1161/ CIRCULATIONAHA.109.192627, indexed in Pubmed: 19704096.

14. Malik VS, Schulze MB, Hu FB, et al. Intake of sugar-sweetened beverages and weight gain: a systematic review. Am J Clin Nutr. 2006; 84(2): 274-288, indexed in Pubmed: 16895873.

15. Kłosiewicz-Latoszek L., Cybulska B. Cukier a ryzyko otyłości, cukrzycy i chorób sercowo-naczyniowych. Probl Hig Epidemiol. 2011; 92(2): 181-186.

16. Schulze MB, Manson JE, Ludwig DS, et al. Sugar-sweetened beverages, weight gain, and incidence of type 2 diabetes in young and middle-aged women. JAMA. 2004; 292(8): 927-934, doi: 10.1001/jama.292.8.927, indexed in Pubmed: 15328324.

17. Guare JC, Wing RR, Grant A, et al. Comparison of obese NIDDM and nondiabetic women: short- and long-term weight loss. Obes Res. 1995; 3(4): 329-335, indexed in Pubmed: 8521149.

18. Pascale RW, Wing RR, Butler BA, et al. Effects of a behavioral weight loss program stressing calorie restriction versus calorie plus fat restriction in obese individuals with NIDDM or a family history of diabetes. Diabetes Care. 1995; 18(9): 1241-1248, indexed in Pubmed: 8612437.

19. Wing RR, Marcus MD, Salata R, et al. Effects of a very-low-calorie diet on long-term glycemic control in obese type 2 diabetic subjects. Arch Intern Med. 1991; 151(7): 1334-1340, indexed in Pubmed: 2064484

20. Wing RR, Blair E, Marcus $M$, et al. Year-long weight loss treatment for obese patients with type II diabetes: does including an intermittent very-low-calorie diet improve outcome? Am J Med. 1994; 97(4): 354-362, indexed in Pubmed: 7942937.

21. Chiasson JL, Josse RG, Gomis R, et al. STOP-NIDDM Trail Research Group, STOP-NIDDM Trail Research Group. Acarbose for prevention of type 2 diabetes mellitus: the STOP-NIDDM randomised trial. Lancet. 2002; 359(9323): 2072-2077, doi: 10.1016/S01406736(02)08905-5, indexed in Pubmed: 12086760

22. Matsuda $M$, DeFronzo RA, Matsuda $M$, et al. Insulin sensitivity indices obtained from oral glucose tolerance testing: comparison with the euglycemic insulin clamp. Diabetes Care. 1999; 22(9): 1462-1470, indexed in Pubmed: 10480510. 